\title{
Joseph F. C. DiMento et Cliff Ellis, Changing lanes. Visions and histories of urban freeways
}

Cambridge (Mass.), The MIT Press, 2013

\section{Konstantinos Chatzis}

\section{(2) OpenEdition Journals}

Édition électronique

URL : http://journals.openedition.org/artefact/729

DOI : 10.4000/artefact.729

ISSN : 2606-9245

\section{Éditeur :}

Association Artefact. Techniques histoire et sciences humaines, Presses universitaires du Midi

\section{Édition imprimée}

Date de publication : 15 juin 2017

Pagination : 218-220

ISBN : 978-2-7535-6525-8

ISSN : 2273-0753

\section{Référence électronique}

Konstantinos Chatzis, " Joseph F. C. DiMento et Cliff Ellis, Changing lanes. Visions and histories of urban freeways », Artefact [En ligne], 5 | 2016, mis en ligne le 15 novembre 2017, consulté le 24 septembre 2020. URL : http://journals.openedition.org/artefact/729; DOI : https://doi.org/10.4000/artefact.729

Ce document a été généré automatiquement le 24 septembre 2020.

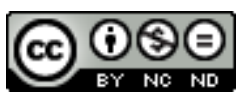

Artefact, Techniques, histoire et sciences humaines est mise à disposition selon les termes de la Licence Creative Commons Attribution - Pas d'Utilisation Commerciale - Pas de Modification 4.0 International. 


\section{Joseph F. C. DiMento et Cliff Ellis, Changing lanes. Visions and histories of urban freeways}

Cambridge (Mass.), The MIT Press, 2013

\section{Konstantinos Chatzis}

\section{RÉFÉRENCE}

Joseph F. C. DiMento et Cliff Ellis, Changing lanes. Visions and histories of urban freeways, Cambridge (Mass.), The MIT Press, 2013, 361 p.

1 En 1993, quelque 1500 personnes se sont réunies pour célébrer l'ouverture de Century Freeway dans l'aire métropolitaine de Los Angeles, à coup sûr la plus chère des autoroutes urbaines dont la nation américaine s'est dotée au $\mathrm{xx}^{\mathrm{e}}$ siècle - moins de vingt miles d'asphalte contre plus de deux milliards de dollars. D'un accouchement difficile - les premières études pour sa localisation datent de l'année 1959 alors que la construction démarre seulement en $1981-$, Century Freeway incorpore plusieurs épisodes de l'histoire des autoroutes urbaines aux États-Unis. Comme pour une bonne partie du système dont elle fait partie, on pense à sa construction dans une atmosphère où l'enthousiasme et l'optimisme dominent : nombreux sont alors ceux qui croient que l'urban freeway est capable non seulement d'assurer les déplacements urbains des citadins américains avec célérité et sécurité, mais qu'elle peut aussi revitaliser une downtown en perte de vitesse, pour des raisons de congestion entre autres, tout en participant à des opérations de slum clearances dans les parties centrales de la ville. Mais, alors que la plupart des autoroutes urbaines ont vu leur construction terminée avant qu'une vague critique à l'égard de ce dispositif technique ne gagne des pans entiers de la population américaine dans les années 1960, Century Freeway est devenue réalité à peine quelques années avant que les premières opérations de démantèlement (removal) de portions d'autoroutes existantes - via leur transformation en boulevards 
urbains ou par l'entremise de leur couverture et la végétalisation de la partie couverte - ne commencent à voir le jour au seuil du xxie siècle.

Changing lanes: Visions and histories of urban freeways, auquel j'emprunte les informations précédentes, est dédié à tous les frères et sœurs de Century Freeway qui quadrillent le territoire américain, presque tous issus du plus grand programme de travaux publics jamais entrepris aux États-Unis, le Dwight D. Eisenhower National System of Interstate and Defense Highways, plus connu comme Interstate Highway System. C'est en 1956 que la décision de construire 41000 miles d'autoroutes reliant les grandes villes du pays, dont 5500 en milieu urbain, est prise; au début des années 2010, le réseau en place ne compte pas moins de 43000 miles $^{1}$.

3 Vue l'ampleur du programme et de ses effets sur la ville et la société américaines, l'objet Interstate System et son histoire ont suscité beaucoup d'intérêt parmi les historiens et autres praticiens des sciences humaines. Le livre signé par DiMento et Ellis, centré sur la partie urbaine du système, est l'un des derniers rejetons de cette littérature foisonnante.

4 Les deux auteurs ont décidé de transporter le lecteur loin dans le temps : on voyage de l'entre-deux-guerres - époque où l'idée de l'urban freeway apparaît et obtient quelques (modestes) réalisations, sous la forme d'urban parkways notamment, à l'instar des constructions conçues et réalisées par Robert Moses dans la région de New York dans les années 1930 - à nos jours. Pour raconter l'histoire de leur sujet, les auteurs s'adonnent aussi à un jeu d'échelles.

5 Ainsi, les cinq premiers chapitres, ordonnés chronologiquement, adoptent une vue panoramique : c'est l'ensemble du territoire urbain des États-Unis qui est couvert par leur étude. Les auteurs identifient les grandes catégories d'acteurs impliqués, de façon asymétrique il est vrai, dans la naissance et la mise en place de l'Interstate System; ils scrutent leurs visions (souvent) contradictoires sur ce qu'est une good city et sur le rôle que l'urban freeway est destinée à jouer dans sa fabrication: défilent ainsi des ingénieurs et des urbanistes, des aménageurs et des architectes, des paysagistes et des fonctionnaires, des hommes politiques enfin. Ces visions, et les actions qui en découlent, sont soigneusement placées dans des contextes (culturels, institutionnels ou financiers) plus larges. Alors que, pendant longtemps, l'autoroute urbaine bénéficie d'un environnement favorable à son déploiement, elle est obligée, à partir des années 1960, d'évoluer sur un terrain de plus en plus miné. En plusieurs endroits, des citadins s'insurgent contre un dispositif qui, d'après les protestataires, défigure leurs villes en éventrant des quartiers entiers, en disloquant des vies communautaires, en obligeant des populations entières, le plus souvent pauvres, à quitter leurs maisons dans le cas de Century Freeway, quelque 21000 personnes ont été déplacées (p. 203). Ces révoltes contre l'autoroute urbaine peuvent s'appuyer, par ailleurs, sur un cadre législatif qui se met en place à partir de la fin des années 1960 et que le «National Historic Preservation Act» (1966) ou l'amendement du «Clean Air Act» (1970) illustrent avec force.

On change d'échelle avec le sixième et dernier chapitre de l'ouvrage. Les auteurs se centrent sur trois projets d'autoroutes urbaines qui ont concerné respectivement les villes de Syracuse (dans l'État de New York), de Memphis (Tennessee) et de Los Angeles. Si chaque cas met en évidence des tendances générales observées au niveau fédéral et à celui des États fédérés, le jeu local des acteurs impliqués, les citadins y compris, dans les trois cas étudiés fait que le résultat final est chaque fois différent. 
7 S'appuyant sur une bibliographie abondante - celle-ci occupe 64 pages (p. 283-346) sans éviter néanmoins quelques omissions ${ }^{2}-$, les auteurs ont écrit une histoire dont ils se veulent à la fois les chroniqueurs fidèles et les témoins engagés. S'ils saluent le fait que l'Interstate Sytem a permis de relier les villes américaines entre elles, ils regrettent l'intrusion de l'autoroute dans l'espace urbain, et ils auraient nettement préféré, à la place de l'automobile, un modèle de déplacements urbains davantage axé sur les transports collectifs. C'est ce parti-pris qui explique peut-être le traitement rapide d'un certain nombre de questions, voire l'existence de blancs dans la narration. Ainsi, peu d'informations sont prodiguées au sujet de l'évolution du parc automobile dans les villes américaines et sur le degré d'efficacité du système autoroutier en matière de vitesse de déplacement et de sécurité, par exemple. Si, d'après les auteurs, ce sont les highway engineers qui ont (malheureusement) imposé leur vision d'urban freeway comme un «tuyau » maximisant le débit des voitures, très peu de choses sont dites sur leurs pratiques $^{3}$. Une question importante, qui traverse néanmoins l'ouvrage, n'est pas traitée de front non plus : à supposer que la good city que les auteurs appellent de leurs vœux soit celle qui accorde une place de choix aux transports collectifs, au vélo et à la marche à pied, que faire si les citadins-citoyens optent, par leurs pratiques effectives de mobilité, pour des formes urbaines basées sur l'usage intensif de la voiture?

Terminons ce compte rendu en invitant le lecteur, déjà familier du sujet ou simple honnête homme, à lire Changing lanes: il y découvrira une bonne introduction, qui greffe sur une synthèse réussie de la littérature existante une prise de position argumentée, à l'histoire d'un objet avec lequel des millions des personnes ont eu, et continueront à entretenir pour des nombreuses années encore, un rapport charnel.

\section{NOTES}

1. Carl Аввотт, Urban America in the Modern Age: 1920 to the Present, Wheeling (IL.), Harlan Davidson, Inc., 2007 ( $2^{\mathrm{e}}$ éd.), p. 83 ; Raymond A. MoHL et Mark H. RoSE, « The Post-Interstate Era: Planning, Politics, and Policy since the 1970s ", Journal of Planning History, vol. 11, n 1, 2012, p. 3.

2. Par exemple, les deux ouvrages suivants ne sont pas cités : Peter D. NORTON, Fighting traffic: the dawn of the motor Age in the American city, Cambridge (Mass.), The MIT Press, 2008 ; Cotten SEILER, Republic of drivers: a cultural history of automobility in America, Chicago, The University of Chicago Press, 2008.

3. Si l'ouvrage signé par David Boyce, Huw Williams, Forecasting urban travel: Past, Present and Future, Cheltenham, Edward Elgar Publishing, 2015, n'était pas disponible au moment de la parution de Changing lanes, les auteurs auraient pu renvoyer aux articles déjà publiés par ces auteurs. 


\section{AUTEURS}

KONSTANTINOS CHATZIS

IFSTTAR 